\title{
The potential role of cell adhesion molecules in the pathogenesis of diabetic neuropathy
}

\author{
E.B. Jude ${ }^{1}$, C. A. Abbott ${ }^{1}$, M. J. Young ${ }^{1}$, S. G. Anderson ${ }^{2}$, J. T. Douglas ${ }^{3}$, A. J. M. Boulton ${ }^{1}$ \\ ${ }^{1}$ Department of Medicine/Diabetes, Manchester Royal Infirmary, Manchester, UK \\ ${ }^{2}$ School of Epidemiology and Health Sciences, University of Manchester, UK \\ ${ }^{3}$ Department of Vascular Biology, Manchester Royal Infirmary, Manchester, UK
}

Summary Cross-sectional studies have shown plasma cell adhesion molecules (CAMs) to be increased in patients with diabetes-related complications. In the first prospective study of CAMs, we have shown that plasma CAMs may be a predictor of the development of diabetic neuropathy. We followed up 28 diabetic patients (13 neuropathic) over a 5 year period, starting from 1991. All patients had peroneal nerve conduction velocity (PNCV), vibration perception threshold and plasma CAMs measured at baseline and follow-up. We found P-selectin and intercellular adhesion molecule -1 (ICAM-1) to be increased at baseline in patients with neuropathy compared to non-neuropathic patients. P-selectin and Eselectin were also found to be significantly higher at baseline in patients who at follow-up showed deterioration in PNCV of more than $3 \mathrm{~m} / \mathrm{s} \quad(p<0.05$; $p=0.01 ;$ respectively). P-selectin and ICAM-1 strongly correlated with PNCV. Univariate and multivariate regression analyses showed a significant inverse association between increasing log P-selectin, $\log$ E-selectin and $\log$ ICAM-1 with decreasing PNCV, and remained significant even after adjustment for glycaemic control. P-selectin and E-selectin, odds ratios of 8.8 (95\%CI: $1.1-68.8 ; p=0.038)$ and 12.5 (95\% CI: $1.2-132.1 ; p=0.036)$, respectively, were significantly associated with the risk of deterioration of PNCV after 5 years. This study suggests that plasma cell adhesion molecules may play an important role in the development and progression of peripheral neuropathy in diabetes mellitus. [Diabetologia (1998) 41: 330-336]

Keywords Diabetes mellitus, cell adhesion molecules, haemostatic factors, nerve conduction velocity, diabetic peripheral neuropathy.
Peripheral neuropathy is one of the commonest complications of diabetes mellitus with a prevalence of around $30-45 \%$ [1-3]. The majority of patients will have developed neuropathy 10-20 years after the onset of diabetes [4] and it is an important cause of foot ulceration, Charcot joints and amputation, with in-

Received: 15 July 1997 and in revised form: 13 October 1997

Corresponding author: Dr. E. B. Jude, Clinical Research Fellow, M7 Records, Department of Medicine, Manchester Royal Infirmary, Oxford Road, Manchester M13 9WL, UK

Abbreviations: CAMs, Cell adhesion molecules; ICAM-1, intercellular adhesion molecule-1; VCAM-1, vascular cell adhesion molecule-1; PAI-1, plasminogen activator inhibitor-1; t$\mathrm{PA}$, tissue plasminogen activator; $\mathrm{PNCV}$, peroneal nerve conduction velocity; VPT, vibration perception threshold. creasing morbidity and mortality. There is therefore an urgent need for prevention of diabetic neuropathy and its sequelae.

The exact pathogenesis of diabetic neuropathy is unclear, with both metabolic and vascular factors having been implicated [5]. Microvascular disease may play an important role in the causation and exacerbation of diabetic neuropathy [6]. Histopathological studies have shown thickening of endoneurial and perineurial vessel walls, hyperplasia of capillary endothelial cells and increased plugging of vessels in neuropathic patients [7-9].

Cell adhesion molecules (CAMs) have been demonstrated to be increased in diabetes mellitus, both insulin-dependent (IDDM) and non-insulin-dependent (NIDDM) [10-13]. They have been implicated in the development of microvascular complications 
Table 1. Characteristics and nerve conduction velocity in diabetic patients with (DN) and without (DNN) neuropathy at baseline

\begin{tabular}{lll}
\hline & $\mathrm{DN}$ & $\mathrm{DNN}$ \\
\hline$n$ & 13 & 15 \\
Age (years) & $53.7 \pm 8.9$ & $45.6 \pm 7.9^{\mathrm{a}}$ \\
$\begin{array}{l}\text { Duration of diabetes } \\
\text { (years) }\end{array}$ & $13.2 \pm 12.3$ & $18.3 \pm 13.3$ \\
IDDM/NIDDM & $7 / 6$ & $6 / 9$ \\
$\left.\mathrm{BMI}^{\mathrm{k} g} / \mathrm{m}^{2}\right)$ & $27.5 \pm 4.8$ & $26.4 \pm 5.5$ \\
$\mathrm{HbA}_{1}(\%)$ & $9.2 \pm 1.5$ & $9.7 \pm 1.8$ \\
Cholesterol & & \\
$(\mathrm{mmol} / \mathrm{l})$ & $6.1 \pm 1.1$ & $5.2 \pm 0.9$ \\
Insulin (pmol/l) & $74.8(31.2-179.4)$ & $78.7(37.1-167.1)$ \\
PNCV (m/s) & $34.4(32.4-36.4)$ & $45.5(41.5-49.9)^{\mathrm{b}}$ \\
VPT (v) & $28.2(20.1-39.6)$ & $14.2(11.5-17.6)^{\mathrm{c}}$ \\
\hline
\end{tabular}

Results given as mean \pm SD or geometric mean (95\% confidence interval)

${ }^{\mathrm{a}} p<0.05,{ }^{\mathrm{b}} p<0.001,{ }^{\mathrm{c}} p<0.01$, for $\mathrm{DN}$ vs $\mathrm{DNN}$

of diabetes. Cross-sectional studies have shown elevated levels of CAMs in patients with diabetic retinopathy [14] and nephropathy [15]. To date no studies have shown CAMs to be a possible aetiological factor in the pathogenesis of diabetic neuropathy. This prospective study was performed to evaluate the role of CAMs in the development and progression of peripheral neuropathy in diabetes.

\section{Subjects and methods}

Patients. Patients recruited into the study were attending the Manchester Diabetes Centre. None of the patients we asked to participate in the study had any diabetic complications, except neuropathy. In 1991, 34 patients were entered into the prospective study; 5 years later 28 diabetic patients (13 IDDM and 15 NIDDM) were re-evaluated. We excluded 6 patients from the final analysis ( 4 patients had moved away, 1 patient refused to attend and 1 patient had died). All participants had a detailed history and physical examination with particular reference to complications of diabetes. The study was approved by the local ethics committee and all patients gave written consent before participating in the study.

Assessment for neuropathy. The following criteria were used for the diagnosis of neuropathy: (i) peroneal nerve conduction velocity (PNCV) under $40 \mathrm{~m} / \mathrm{s}$ and (ii) vibration perception threshold (VPT) of 2 standard deviations or more than the normal age-related mean [16, 17].

We measured PNCV with standard surface electrodes using a Medelec (Medelec, Woking, UK) neurophysiology system for all recordings. Nerve stimulation was performed proximally at the head of the fibula and distally on the shin, $10-15 \mathrm{~cm}$ above the ankle, with the recording electrode over the extensor digitorum brevis on the lateral border of the foot. All stimulations were performed using a supramaximal stimulus and an average of 30 stimulations was used in severely neuropathic patients when the action potential was reduced. Tracings were taken of the motor action potentials and the latencies were measured by an independent observer without knowledge of the subject's identity. Patients were designated as neuropathic if they had a mean PNCV of less than $40 \mathrm{~m} / \mathrm{s}[16,17]$.
Vibration perception threshold (VPT) was measured using a biothesiometer (Biomedical Instrument Co, Newbury, Ohio, USA). A minimum of three readings were taken on the big toe bilaterally. VPT was then recorded as a mean of both feet.

Assessment of other complications. The subjects had lying blood pressure (BP), measured twice, in the right arm using Dinamap (Critikon, Johnson \& Johnson Medical, Ascot, Berkshire, UK), after resting for $10 \mathrm{~min}$. Hypertension was defined according to World Health Organisation criteria (systolic $\mathrm{BP}>160 \mathrm{mmHg}$ and /or diastolic $\mathrm{BP}>95 \mathrm{mmHg}$ ) or if the patient was on current antihypertensive treatment. BMI was calculated by weight $(\mathrm{kg}) /$ height $\left(\mathrm{m}^{2}\right)$. Direct ophthalmoscopy for retinopathy was performed through dilated pupils. Microalbuminuria (overnight albumin excretion rate of more than $20 \mu \mathrm{g} / \mathrm{min}$ ) was measured by two timed overnight collections of urine.

Coronary artery disease was determined by 12 lead ECG (using Minnesota code) [18], hospital records of confirmed myocardial infarction, definite history of angina or coronary artery bypass grafting. Cerebrovascular disease was assessed by history and clinical examination and hospital records of definite stroke. Peripheral vascular disease was determined by history of intermittent claudication and ankle brachial pressure index (normal $>0.9$ ) using a multi dopplex machine (Huntleigh Nesbit Evans Healthcare, Cardiff, UK).

Patients with hypertension, retinopathy, microalbuminuria, manifest macrovascular disease or taking aspirin, non-steroidal anti-inflammatory drugs, evening primrose oil, vasoactive medication (such as beta-blockers), calcium antagonists and angiotensin converting enzyme (ACE) inhibitors were excluded from the study. None of the subjects smoked.

Biochemical analysis. Blood from an antecubital vein was collected after an overnight fast and after resting for $15 \mathrm{~min}$ in the supine position. All patients had fasting glucose (Glucose oxidase; Boehringer Mannheim, Mannheim, Germany), glycated haemoglobin (normal $<8 \%$ ) (HPLC, Diamat; BioRad, Hercules, Calif., USA), serum creatinine and cholesterol (Hitachi 747, Hitachi, Japan)and microalbuminuria (Immunonephelometry, Array Protein system, Beckman Instruments Inc., Brea, Calif., USA) concentrations estimated at baseline and follow-up. Plasma for CAMs, plasminogen activator inhibitor1 (PAI-1), tissue-plasminogen activator (t-PA) and serum insulin was separated after centrifugation at $3000 \mathrm{rev} / \mathrm{min}$ at $4{ }^{\circ} \mathrm{C}$ for $20 \mathrm{~min}$ and plasma obtained stored at $-70^{\circ} \mathrm{C}$ until analysis. CAMs (P-selectin, E-selectin, ICAM-1 and VCAM-1; interassay coefficients of variation $<9 \%$ ) were determined using standard immunoassay kits (R\&D Systems Europe, Abingdon, Oxford, UK). We measured t-PA activity and PAI-1 activity to assess the fibrinolytic process by chromogenix assay using standard commercial test kits (Coatest PAI and Coaset t-PA respectively; Chromogenix AB, Molndal, Sweden). The interassay coefficients of variation were $6 \%$ for both the t-PA and PAI- 1 assays. Plasma fibrinogen was measured using Clauss assay (ACL 300R; IL. Instruments, Warrington, UK); interassay coefficient of variation less than $6 \%$.

At follow-up, the clinical examination, 12-lead ECG, ankle brachial pressure index, VPT and PNCV were performed in all patients using the same instruments and procedures as described above.

Statistical methods. Results are presented as mean \pm SD. Geometric means (95\% confidence interval) are presented for PNCV, VPT, insulin, haemostatic factors and CAMs because the underlying data were skewed. Unpaired $t$-tests, two-sided, were used to assess significant difference in means between 
Table 2. Characteristics of diabetic patients with (A) and without (B) decreasing peroneal nerve conduction velocity $(\mathrm{PNCV})^{\mathrm{a}}$ at follow-up

\begin{tabular}{lll}
\hline & A & B \\
\hline$n$ & 14 & 14 \\
$\mathrm{BMI}\left(\mathrm{kg} / \mathrm{m}^{2}\right)$ & $27.1 \pm 5.4$ & $26.9 \pm 4.6$ \\
$\mathrm{HbA}_{1}(\%)$ & $9.3 \pm 1.4$ & $9.6 \pm 2.0$ \\
$\begin{array}{l}\text { Cholesterol } \\
(\mathrm{mmol} / \mathrm{l})\end{array}$ & $6.2 \pm 1$ & $5.2 \pm 1.1$ \\
Microalbuminuria & & \\
( $\mu \mathrm{g} /$ min) & $17.8(6.9-45.8)$ & $18.1(5.8-56.7)$ \\
Retinopathy & 9 & 2 \\
Macrovascular disease & 6 & 5 \\
Coronary artery disease & 4 & 3 \\
Stroke & 1 & 1 \\
Peripheral vascular disease & 1 & 1 \\
PNCV (m/s) & $33.5(28.4-39.9)$ & $38.1(36.7-41.5)^{\mathrm{b}}$ \\
VPT (v) & $27.7(20.9-36.8)$ & $21.3(14.5-31.1)$ \\
\hline
\end{tabular}

Results given as mean \pm SD or geometric mean $(95 \%$ confidence interval)

${ }^{a}$ Decreasing PNCV defined as: Decrease in NCV $>3 \mathrm{~m} / \mathrm{s}$

${ }^{\mathrm{b}} p<0.05$ for group A vs B

groups. Spearman's rank correlations were used to measure associations between various parameters. Univariate and multivariate linear analyses were used to assess the relationship between PNCV as a continuous variable and the log concentrations of CAMs. Logistic regression analyses were used to assess the relationship between patients who showed a deterioration of PNCV more than $3 \mathrm{~m} / \mathrm{s}$ and the concentration of the CAMs which were $\log$ transformed before analysis. All analyses were performed using SPSS for Windows 6.3 (SPSS Inc., Chicago, Illinois, USA) and Intercooled Stata 5.0 (College Station, Texas, USA).

\section{Results}

We entered 28 diabetic patients (13 IDDM and 15 NIDDM) into the final analysis in this 5 year prospective study. Of the patients 13 had neurophysiological evidence of neuropathy at baseline. Patients with neuropathy were older but there was no difference in duration of diabetes, $\mathrm{BMI}, \mathrm{HbA}_{1}$, cholesterol and serum insulin concentrations between the two groups (Table 1). VPT was significantly higher in the neuropathic patients at baseline and follow-up.

PNCV [geometric mean (95\% CI)] was significantly lower in neuropathic patients compared to non-neuropathic diabetic patients at baseline [34.4 (32.4-36.4) vs $45.5(41.5-49.9) \mathrm{m} / \mathrm{s} ; p<0.0001]$ and at follow-up [31.6 (26.8-37.3) vs $41.1(39.5-42.8) \mathrm{m} /$ $\mathrm{s} ; \quad p<0.001]$. In the non-neuropathic patients PNCV was $45.5(41.5-49.9) \mathrm{m} / \mathrm{s}$ and 41.1 (39.5$42.8) \mathrm{m} / \mathrm{s}$ at baseline and follow-up respectively $(p<0.05)$. Therefore, mean reduction in PNCV in non-neuropathic patients was $4.4 \mathrm{~m} / \mathrm{s}$ or $0.9 \mathrm{~m} \cdot \mathrm{s}^{-1}$ $\cdot$ year $^{-1}$.

Deterioration in nerve function Patients were regrouped with reference to deterioration in the PNCV by $3 \mathrm{~m} / \mathrm{s}$ over the 5 years. Group A consisted of 14 patients $(50 \%)$ who showed a reduction in PNCV of over $3 \mathrm{~m} / \mathrm{s}$; and group B consisted of 14 patients who did not. Eight patients in group A and 5 in group B had neuropathy at baseline. No difference was found in age, duration of diabetes, BMI, $\mathrm{HbA}_{1}$, cholesterol and insulin concentrations at baseline between the two groups. Although PNCV was not different at baseline between group A and B [39.9 (33.9-44.6) vs $38.9(36.5-41.5) \mathrm{m} / \mathrm{s} ; p=0.12]$, it was significantly lower in group A at follow-up [33.5 (28.4-39.9) vs $38.1(36.7-41.5) \mathrm{m} / \mathrm{s} ; p=0.04]$ (Table 2). Of the 15 non-neuropathic patients 4 were found to have developed neuropathy at follow-up. VPT was higher in group A compared to group B at baseline and follow-up, but not significantly so. This could be explained by the fact that the biothesiometer has an upper limit and many patients with neuropathy had VPT which was above the recordable limit of the instrument .

None of the patients had retinopathy at baseline. At the end of the study 11 patients (6 IDDM) were found to have developed retinopathy of whom 6 patients had neuropathy at baseline. Nine of these patients showed reduction in PNCV of more than $3 \mathrm{~m} / \mathrm{s}$.

At follow-up 7 patients were diagnosed with coronary artery disease, 1 patient had suffered a stroke and and 1 patient has multiple transient ischaemic at-

Table 3. Cell adhesion molecules and haemostatic factors in diabetic patients with (DN) and without (DNN) neuropathy at baseline.

\begin{tabular}{lll}
\hline & DN & DNN \\
\hline P-selectin (ng/ml) & $176.5(134.7-231.2)$ & $136.2(111.1-166.9)^{\mathrm{a}}$ \\
E-selectin (ng/ml) & $50.4(38.9-65.2)$ & $40.4(30.9-52.7)$ \\
ICAM-1 (ng/ml) & $278.4(249.0-311.2)$ & $228.17(199.82-260.54)^{\mathrm{b}}$ \\
VCAM-1 (ng/ml) & $600.9(490.9-735.6)$ & $549.7(465.1-649.5)$ \\
PAI-1 activity (IU/l) & $5.3(2.0-14.2)$ & $8.0(3.5-18.7)$ \\
t-PA activity (IU/l) & $0.34(0.17-0.67)$ & $0.46(0.2-1.03)$ \\
Fibrinogen (g/l) & $3.94(3.6-4.4)$ & $2.95(2.5-3.5)^{\mathrm{c}}$ \\
\hline
\end{tabular}

Results given as geometric mean (95\% confidence interval)

${ }^{\mathrm{a}} p<0.05 ;{ }^{\mathrm{b}} p=0.01 ;{ }^{\mathrm{c}} p<0.01$, DN vs DNN 
Table 4. Baseline cell adhesion molecules and haemostatic factors in diabetic patients with (A) and without (B) decreasing peroneal nerve conduction velocity at follow-up

\begin{tabular}{lll}
\hline & $\mathrm{A}$ & $\mathrm{B}^{\mathrm{a}}$ \\
\hline P-selectin $(\mathrm{ng} / \mathrm{ml})$ & $184.8(149.2-228.9)$ & $129.1(99.9-167.0)^{\mathrm{a}}$ \\
E-selectin $(\mathrm{ng} / \mathrm{ml})$ & $54.7(44.0-68.0)$ & $36.7(27.9-48.2)^{\mathrm{b}}$ \\
ICAM-1 $(\mathrm{ng} / \mathrm{ml})$ & $255.4(219.9-296.6)$ & $252.0(225.7-281.4)$ \\
VCAM-1 $(\mathrm{ng} / \mathrm{ml})$ & $592.7(503.2-698.2)$ & $558.4(448.2-695.8)$ \\
PAI-1 activity (IU/l) & $3.8(1.3-10.7)$ & $11.8(7.2-19.5)$ \\
t-PA activity (IU/l) & $0.33(0.15-0.70)$ & $0.47(0.24-0.94)$ \\
Fibrinogen $(\mathrm{g} / \mathrm{l})$ & $3.7(3.2-4.3)$ & $3.2(2.8-3.8)$ \\
\hline
\end{tabular}

Results given as geometric mean (95\% confidence interval)

${ }^{\mathrm{a}} p<0.05 ;{ }^{\mathrm{b}} p=0.01$ Group A vs B

Table 5. Linear regression of baseline cell adhesion molecules and peroneal nerve conduction velocity in diabetic patients and relationship with $\mathrm{HbA}_{1}$

\begin{tabular}{lllll}
\hline & & $\beta$-coefficient & $R^{2}$ & $p$-value \\
\hline P-selectin & $\mathrm{A}$ & -0.26 & 0.24 & 0.02 \\
& $\mathrm{~B}$ & -0.31 & 0.32 & 0.02 \\
E-selectin & $\mathrm{A}$ & -0.25 & 0.25 & 0.01 \\
& $\mathrm{~B}$ & -0.29 & 0.39 & 0.005 \\
ICAM-1 & $\mathrm{A}$ & -0.52 & 0.27 & 0.007 \\
& $\mathrm{~B}$ & -0.49 & 0.24 & 0.04 \\
VCAM-1 & $\mathrm{A}$ & -0.026 & 0.001 & 0.86 \\
& $\mathrm{~B}$ & -0.01 & 0.001 & 0.94
\end{tabular}

$\mathrm{A}$, Before and B, after adjustment for $\mathrm{HbA}_{1}$

tacks. Two patients were found to have peripheral vascular disease (Table 2).

Haemostatic factors and CAM. Plasma P-selectin [176.5 (134.7-231.2) vs 136.2 (111.1-166.9) ng/ml; $p<0.05)$ and ICAM-1 [278.4 (249.0-311.2) vs 228.17 (199.8-260.5) ng/ml; $p=0.019]$ concentrations at baseline were significantly higher in diabetic patients with neuropathy than those without (Table 3). E-selectin and VCAM-1 although elevated in patients without neuropathy were not significantly different between the two groups. t-PA and PAI-1 activity were similar in diabetic subjects with and without neuropathy. However, fibrinogen levels were significantly higher in the neuropathic compared to nonneuropathic patients [3.94 (3.6-4.4) vs 2.95 (2.5-3.5), $\mathrm{g} / \mathrm{l} ; p<0.01]$.

Plasma P-selectin [184.8 (149.2-228.9) vs 129.1 (99.9-167.0) $\mathrm{ng} / \mathrm{ml} ; p<0.05]$ and E-selectin [54.7 (44.0-68.0) vs 36.7 (27.9-48.2); $p=0.01]$ were significantly elevated in patients who showed a deterioration of PNCV of more than $3 \mathrm{~m} / \mathrm{s}$ (group A) compared to group B (Table 4). No difference was observed in ICAM-1, VCAM-1, fibrinogen and t-PA and PAI-1 activities between the two groups.

Baseline ICAM-1 and VCAM-1 were found to be weakly correlated with age $(r=0.18$ and 0.23$)$ while E-selectin and P-selectin were negatively correlated $(r=-0.21$ and -0.22$)$, but none of these rela- tionships was significant. Duration of diabetes did not correlate with baseline CAMs. There were no differences in mean CAMs and tPA and PAI-1 activity between IDDM and NIDDM patients at baseline. At follow-up plasma CAMs (except for P-selectin) were higher but not significantly, in the patients with neuropathy. This may be related to the development of macro- and microvascular disease at follow-up.

Correlations between CAMs, haemostatic factors, glycaemic control and PNCV. Correlations were performed with the biochemical parameters measured and NCV at baseline and follow-up. ICAM-1 was strongly correlated with nerve conduction velocity at baseline $(r=-0.594, p=0.002)$ and follow-up $(r=$ $-0.608, p=0.001$ ) (Fig. 1). P-selectin correlated with NCV at baseline $(r=-0.487, p=0.018)$ and at follow-up $(r=-0.494, p=0.012)$ (Fig. 2). There was no correlation between VCAM-1, E-selectin and haemostatic factors and NCV at baseline and follow-up. $\mathrm{HbA}_{1}$ did not show a correlation with worsening nerve conduction velocity, cell adhesion molecules or haemostatic factors.

Regression analysis. Linear regression analyses showed a significant inverse association between increasing concentrations of both $\log \mathrm{P}$-selectin $(\beta=$ $\left.-0.27, \mathrm{r}^{2}=0.24 ; p=0.02\right), \log$ E-selectin $(\beta=-0.25$, $\left.\mathrm{r}^{2}=0.25 ; p=0.01\right)$ and $\log$ ICAM-1 $(\beta=-0.52$, $\left.\mathrm{r}^{2}=0.27 ; p=0.007\right)$ and a decrease in PNCV. These three CAMs remained independently (and significantly) associated with a deterioration of PNCV even after adjusting for glycaemic control (Table 5). The association between log VCAM-1 and PNCV was not statistically significant. Logistic regressions were performed to investigate whether increasing log transformed concentrations of baseline CAMs may be associated with a risk of decreasing PNCV of more than $3 \mathrm{~m} / \mathrm{s}$. P-selectin and E-selectin with odds ratios (OR) of 8.8 (95\% CI: $1.1-68.8 ; p=0.038)$ and 12.5 (95\% CI: 1.2-132.1; $p=0.036$ ), respectively, were significantly associated with the risk of having a change in PNCV after 5 years. This association re- 


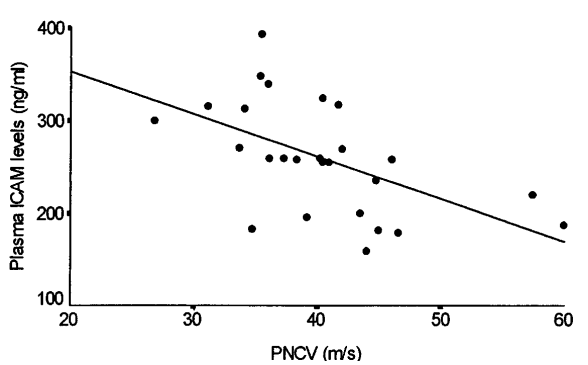

Fig.1. Correlation between peroneal nerve conduction velocity (PNCV) and intercellular adhesion molecule (ICAM-1) at baseline $(r=-0.594 ; p=0.002)$

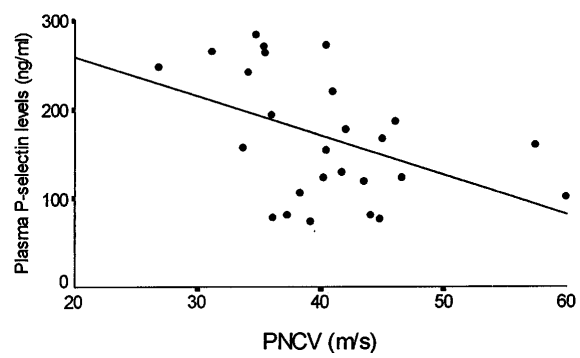

Fig. 2. Correlation between peroneal nerve conduction velocity (PNCV) and P-selectin at baseline $(r=-0.487 ; p=0.018)$

mained significant even after adjusting for $\mathrm{HbA}_{1}$. ICAM-1 and VCAM-1 were not associated with the risk of a deterioration in PNCV.

\section{Discussion}

In this study we have demonstrated that elevated plasma P- and E-selectin levels predict the deterioration of nerve function over 5 years. As the term 'diabetic neuropathy' represents a heterogeneous group of conditions, and it is difficult to define when a patient changes from non-neuropathic to neuropathic [19], the most sensitive measure of nerve function, $\mathrm{NCV}$, was used as a marker. We have previously confirmed that peroneal NCV is the best surrogate marker for nerve function in that it is reproducible, correlates with nerve structure, predicts the development of neuropathy $[19,20]$ and is associated with the endpoint of the condition, insensitive foot ulceration [21,22]. Thus, at follow-up, loss of nerve function as measured by a reduction in PNCV was used as a measure of deterioration across the whole group, rather than splitting the group into neuropathic and nonneuropathic by the use of pre-defined and potentially artificial criteria.

Increased levels of circulating CAMs have been observed in diabetes, even in the absence of diabetic complications $[11,12]$. CAMs have also been implicated in the development of microangiopathy and increased levels have been demonstrated in diabetic patients with long-term complications. Increased VCAM-1 levels have been observed in IDDM patients with diabetic retinopathy and microalbuminuria compared to those without these complications [14, 15]. McLeod et al. [23] reported increased expression of ICAM-1 and P-selectin in the arteries and veins of the retinal vasculature in diabetic patients [23]. In our prospective study we have shown increased levels of P-selectin and ICAM-1 in patients with neuropathy and have demonstrated that increased P-selectin and E-selectin at baseline predicted worsening of neuropathy in our cohort of diabetic patients.

P, E and L-selectins and ICAM-1 and VCAM-1 constitute the CAMs. CAMs are expressed by activated endothelium and shed into the circulation [24], and increased circulating levels which can be measured in the plasma have been observed in many disease states [25]. CAMs have been shown to be involved in the interaction between circulating leucocytes and the endothelium. Increased expression of CAMs enhances the "stickiness" of the endothelium to cellular components of the bloodstream, most specifically monocytes [25]. Monocytes are activated by exposure to the increased levels of cytokines in the plasma of diabetic patients [26], resulting in increased expression of growth factors, cytokines and free radicals damaging the endothelium leading to platelet adhesion and thrombus formation as well as stimulating vascular smooth muscle cell proliferation [25]. Histopathological examination of diabetic nerve capillaries has demonstrated occlusion of blood vessels with endothelial cell hyperplasia and thickening of the capillary basement membrane with a reduction in capillary lumen [16].Measurement of nerve oxygen tension in sural nerves of diabetic patients with neuropathy has shown this to be significantly lower when compared to non-neuropathic subjects [27]. Thus, occlusion of the microvasculature with subsequent ischaemia and hypoxia of the peripheral nerves could possibly result in diabetic neuropathy. However, further studies are required to assess the changes caused by CAMs on nerve capillaries.

CAMs are involved in the pathogenesis of atherosclerosis [28]. None of our patients had clinically manifest macrovascular disease at entry into the study. This however does not rule out the possibility of our patients having subclinical macrovascular disease; which also could explain the finding of elevated levels of CAMs. At follow-up an equal number of patients had developed macrovascular disease in groups A and B (Table 2). With the exception of neuropathy, our patients did not have any other diabetic complication at entry into the study. Hence elevated CAMs in neuropathic patients suggests an association of CAMs in its pathogenesis.

Diabetes is known to be associated with endothelial dysfunction and the observation of increased CAMs in the absence of micro- or macrovascular 
complications suggests an activated endothelium in diabetic patients and is associated with release of endothelial products which could possibly be related to the development of microangiopathy [29, 30]. Diabetes is also described as a hypercoagulable state and rheological parameters and endothelial products have been shown to be abnormal in diabetic subjects with microangiopathy [31, 32]. Plater et al. [33] specifically demonstrated elevated von Willebrand factor antigen (vWFag) at baseline as a predictor of deterioration in diabetic nerve function [33], although Ford et al. [16] could not demonstrate elevated vWFag when measured by ELISA. In addition no difference was found in PAI, plasminogen, fibrinogen and plasma viscosity. Similarly, we did not find a difference in fibrinogen and PAI-1 and t-PA activities at baseline in patients with deterioration in NCV as well as, cross-sectionally, in neuropathic patients, except in the latter fibrinogen levels were higher than non-neuropathic patients. No correlation was seen between haemostatic factors and CAMs. This possibly indicates that CAMs act independently of the various haemorrheological parameters in the pathogenesis of microangiopathy and diabetic complications.

Glycaemic control has been shown to influence development of diabetic complications [34], but its effect on CAMs is still unclear [11,12]. In our study glycaemic control was not related to deterioration in diabetic nerve function, although it was only estimated at one point in time after baseline. Neither did glycaemic control correlate with CAM levels, in agreement with the study by Steiner et al. [11]. Thus suggesting that CAMs, especially P-selectin and ICAM1 , may be independent factors in the pathogenesis and progression of diabetic neuropathy. A significant inverse association was shown to be present between P-selectin, E-selectin and ICAM-1 and PNCV even after adjusting for glycaemic control. Thus, whereas strict metabolic control is important in preventing diabetic complications, it may not be totally successful as vascular factors may not always be influenced by glycaemic control.

In conclusion, in the first prospective study of CAMs in the pathogenesis of diabetic complications, we have shown that elevated levels of P-selectin and E-selectin are major risk factors for and predicts deterioration of nerve function in diabetic patients. Secondly, P-selectin and ICAM-1 concentrations were found to be increased in diabetic neuropathic patients. However, larger prospective studies are required to firmly establish a link between plasma CAM levels and the development of diabetic peripheral neuropathy.

Acknowledgement. EB Jude is supported by a grant from the British Diabetic Association

\section{References}

1. Young MJ, Boulton AJM, McLeod AF, Williams DRR, Sonksen PH (1993) A multicentre study of the prevalence of diabetic neuropathy in the UK hospital clinic population. Diabetologia 36: 150-154

2. Tesfaye S, Stevens LK, Stephenson JM et al. (1996) The prevalence of diabetic peripheral neuropathy and its relation to glycaemic control and potential risk factors: the Eurodiab IDDM complications study. Diabetologia 39: 1377-1384

3. Dyck PJ, Kratz KM, Karnes JL et al. (1993) The prevalence by staged severity of various types of diabetic neuropathy, retinopathy and nephropathy in a population-based cohort: the Rochester diabetic neuropathy study. Neurology 43: 817-824

4. Lee BC, Tooke JE (1997) An overview of diabetic complications. In: Boulton AJM (ed) Diabetic neuropathy. Marius Press, Lancashire, UK, pp 21-39

5. Cotter MA, Cameron NE (1997) The aetiopathogenesis of diabetic neuropathy; Metabolic theories and vascular theories. In: Boulton AJM (ed) Diabetic neuropathy. Marius Press, Lancashire, UK, pp 97-146

6. Tesfaye S, Malik RA, Ward JD (1994) Vascular factors in diabetic neuropathy. Diabetologia 37: 847-853

7. Malik RA, Newrick PG, Sharma AK et al. (1989) Microangiopathy in human diabetic neuropathy: relationship between capillary abnormalities and the severity of neuropathy. Diabetologia 32: 92-102

8. Dyck PJ, Hansen S, Karnes J et al. (1985) Capillary number and percentage closed in human diabetic sural nerve. Proc Natl Acad Sci USA 82: 2513-2517

9. Timperley WR, Boulton AJM, Davies-Jones GA, Jarrett JA, Ward JD (1985) Small vessel disease in progressive diabetic neuropathy associated with good metabolic control. J Clin Pathol 38: 1030-1038

10. Fasching P, Waldhausl W, Wagner OF (1996) Elevated circulating adhesion molecules in NIDDM - potential mediators in diabetic macroangiopathy. Diabetologia 39: 12421244

11. Steiner M, Reinhardt KM, Krammer B, Ernst B, Blann AD (1994) Increased levels of soluble adhesion molecules are independent of glycaemic control. Thromb Haemost 72: 979-984

12. Cominacini L, Pasini AF, Garbin U et al. (1995) Elevated levels of soluble E- selectin in patients with IDDM and NIDDM: relation to metabolic control. Diabetologia 38: $1122-1124$

13. Jilma B, Fasching P, Ruthner C et al. (1996) Elevated circulating P-selectin in insulin dependent diabetes mellitus. Thrombosis Haemostasis 76: 328- 332

14. Fasching P, Veitl M, Rohac M, et al. (1996) Elevated concentrations of circulating adhesion molecules and their association with microvascular complications in insulin-dependent diabetes mellitus. J Clin Endocrinol Metab 81: 4313-4317

15. Schmidt AM, Crandall J, Hori O, Cao R, Lakatta E (1996) Elevated plasma levels of vascular cell adhesion molecule1 (VCAM-1) in diabetic patients with microalbuminuria: a marker of vascular dysfunction and progressive vascular disease. Br J Haematol 92: 747-750

16. Ford I, Malik RA, Newrick PG, Preston FE, Ward JD (1992) Relationships between haemostatic factors and capillary morphology in human diabetic neuropathy. Thromb Haemost 68: 628-633

17. Young MJ, Jones GC (1997) Diabetic neuropathy: symptoms, signs and assessment. In: Boulton AJM (ed) Diabetic neuropathy. Marius Press, Lancashire, UK, pp 41-62 
18. Blackburn H, Keys A, Simonsen E, Rautaharju P, Punsar S (1960) The electrocardiogram in population studies: a classification system. Circulation 21: 1160-1175

19. Veves A, Malik RA, Masson EA, Sharma AK, Schady W, Boulton AJM (1991) The relationship between sural nerve morphometric findings and measures of peripheral nerve function in mild diabetic neuropathy. Diabetic Med 8: 917-921

20. Ziegler D, Mayer P, Muhlen H, Greis FA (1991) The natural history of somatosensory and autonomic nerve dysfunction in relation to glycaemic control during the first 5 years after diagnosis of type 1(insulin-dependent) diabetes mellitus. Diabetologia 34: 822-829

21. Boulton AJM, Hardisty CA, Betts RB et al. (1983) Dynamic foot pressure and other studies as diagnostic and management aids in diabetic neuropathy. Diabetes Care 6: 26-33

22. Young RJ, Zhou YQ, Rodriquez E et al. (1986) Variable relationship between peripheral somatic and autonomic neuropathy in patients with different syndromes of diabetic polyneuropathy. Diabetes 35: 192-197

23. McLeod DS, Lefer DJ, Merges C, Lutty GA (1995) Enhanced expression of intracellular adhesion molecule-1 and $\mathrm{P}$-selectin in the diabetic human retina and choroid. Am J Pathol 147: 642-665

24. Gearing AJH, Newman W (1993) Circulating adhesion molecules in disease. Immunol Today 14: 506-512

25. Faruqi RM, DiCorleto PE (1993) Mechanisms of monocyte recruitment and accumulation. Br Heart J 69[Suppl]:S19S29

26. Yamada K, Naoko T, Otabe S, Inada C, Inoue M, Nonaka K (1993) Pancreatic $\beta$-cell-selective production of tumour necrosis factor- $\alpha$ induced by interleukin-1. Diabetes 42 : 1026-1031

27. Newrick PG, Wilson AJ, Jakubowski J, Boulton AJM, Ward JD (1986) Sural nerve oxygen tension in diabetes. BMJ 293: 1053-1054

28. Poston RN, Dorian OH, Coucher JR, Nichola PG, Johnson-Tidey RR (1994) Expression of intercellular adhesion molecule-1 in atherosclerotic plaques. Am J Pathol 140: 665-673

29. Lorenzi M, Cagliero E (1991) Pathobiology of endothelial and other vascular cells in diabetes mellitus. Call for data. Diabetes 40: 653-659

30. Poston L, Taylor PD (1995) Endothelium-mediated vascular function in insulin-mediated diabetes mellitus. Clin Sci 88: 245-255

31. Young MJ, Bennett JL, Liderth SA, Veves A, Douglas JT, Boulton AJM (1996) Rheological and microvascular parameters in diabetic peripheral neuropathy. Clin Sci 90: 183-187

32. Walker JD, Viberti GC (1991) Pathophysiology of microvascular disease: an overview. In: Pickup J, Williams G (eds) Textbook of diabetes. Blackwell Scientific Publications, Oxford, pp 526-533

33. Plater ME, Ford I, Dent MT, Preston FE, Ward JD (1996) Elevated von Willebrand factor antigen predicts deterioration in diabetic peripheral nerve function. Diabetologia 39: 336-343

34. The Diabetes Control and Complications Trial Research Group (1993) The effect of intensive treatment of diabetes on the development and progression of long-term complications in insulin-dependent diabetes mellitus. N Engl J Med 329: 977-986 